thought to have a role in disease. We aimed to determine pulmonary specific interplay between BMPR2 and IL-1B signalling through assessing IL- $1 \beta$ responsiveness of pulmonary artery and aortic smooth muscle cells (Ao/PA SMC) and determine the effects of reduced BMPR2.

Methods Microarray analysis of PASMC and AoSMC mRNA was performed using microarray on mRNA isolated from cells cultured in SMGM-2 (Lonza) +/- functional BMPR2 (by use of siRNA) and stimulation with $10 \mathrm{ng} / \mathrm{ml} \mathrm{IL-1ß}$ for $6 \mathrm{~h}$. Subsequent bioinformatics was performed using R. Findings were validated using quantitative PCR and western blotting. Furthermore

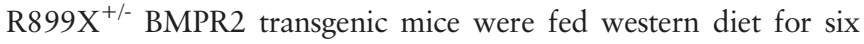
weeks and injected daily with IL-1ß then assessed for inflammatory activation and PAH phenotype (catheter/echo). mRNA and protein changes were measured by TaqMan PCR, western blotting and serum ELISA. Immuno-staining of paraffin embedded lung sections assessed pulmonary vascular remodelling.

Results Array data shows reduced inflammatory activation in response to IL-1ß in PASMC compared with AoSMCs, analysis of cells lacking functional BMPR2 identified an exaggerated inflammatory response to IL- $1 \beta$ in PASMC lacking BMPR2 (siRNA). Significant up-regulation of IL-6, IL-1 $\alpha$ and adhesion molecules ( $>2$-fold) shown by array analysis was validated by qPCR. In the absence of BMPR2 a 1.5 fold increase in proliferation was observed in response to IL- $1 \beta$ compared to PASMC with functional BMPR2. Mice treated with IL-1ß show higher white blood cell counts (1.7-fold), and protein levels of OPG and IL-6 (serum) matching in vitro data.

Conclusion IL-1ß induces a pulmonary specific transcriptome altered by suppression of BMPR2 signalling indicating cross-talk between the pathways. In the presence of BMPR2, PASMCs show limited response to IL-1ß however reducing BMPR2 exacerbated this response increasing the likelihood of a PAH phenotype in PASMCs. This highlights a mechanism that increased IL$1 \beta$ may provide "second hit" to reduced BMPR2 to stimulate development of PAH.

\section{S4 PULMONARY ARTERY PRESSURE AND EXERCISE TOLERANCE IN PATIENTS WITH PULMONARY ARTERIOVENOUS MALFORMATIONS}

${ }^{1} \mathrm{~T}$ Hall, ${ }^{2} \mathrm{H}$ Tighe, ${ }^{2} \mathrm{~K}$ Hornby, ${ }^{2} \mathrm{M}$ Park, ${ }^{1} \mathrm{~V}$ Santhirapala, ${ }^{2} \mathrm{~K}$ Murphy, ${ }^{2} \mathrm{JE}$ Jackson, ${ }^{2} \mathrm{~L}$ Howard, ${ }^{3} \mathrm{CL}$ Shovlin. ${ }^{1}$ Imperial College School of Medicine, London, UK; ${ }^{2}$ Imperial College Healthcare NHS Trust, London, UK; ${ }^{3}$ Imperial College London, London, UK

\subsection{6/thoraxjnl-2015-207770.10}

Introduction and objectives Pulmonary arteriovenous malformations (PAVMs) provide direct communications between pulmonary arteries and pulmonary veins, bypassing the pulmonary capillary bed. Greater improvements in exercise capacity following embolization therapy have been reported by PAVM patients with raised pulmonary artery pressures (PAPs). The objective of this study was to test the hypothesis that lower PAPs are associated with greater exercise capacity in hypoxaemic PAVM patients. Methods Patients attending our tertiary care institution were recruited at the time of PAVM assessment or embolization. Pulmonary artery pressure measurements were obtained by rightheart catheterisation immediately prior to contrast injection and embolization. For both patients and healthy controls, incremental cardiopulmonary exercise tests were performed seated on an adjustable cycle ergometer (MasterScreen CPX; Via Sprint).
Results A total of 19 patients and 26 controls were recruited for the study. Significantly lower arterial oxygen saturations (median $91 \%$ vs. $98 \%, \mathrm{p}<0.0001$ ) and end-tidal $\mathrm{PCO}_{2}$ levels were demonstrated in patients alongside significantly raised $\mathrm{V}$ [dot]e/V[dot] $\mathrm{CO}_{2}$ slopes $(32.2$ vs. $24.1 \mathrm{~L} / \mathrm{min} / \mathrm{L} / \mathrm{min}, \mathrm{p}=0.0003)$. The regression models that best described peak oxygen uptake $(\mathrm{V}$ $\left.[\operatorname{dot}] \mathrm{O}_{2}\right)$ as a percent of predicted values, were similar between PAVM patients and controls. Surprisingly, despite the differences in $\mathrm{SaO}_{2}$ between PAVM and control subjects, the addition of $\mathrm{SaO}_{2}$ to the final models did not improve the significance of the final model nor did it raise the adjusted $-\mathrm{r}^{2}$ value. For the PAVM subgroup, the median mean PAP was $14.5 \mathrm{mmHg}$, (IQR 12.516.0; range 6-22). Univariate and multiple regression analyses demonstrated no significant relationships between systolic, diastolic or mean PAPs, and peak exercise parameters.

Conclusions This study has demonstrated in a population of PAVM patients with hypoxaemia and PAPs essentially within the normal range, that PAP does not have a major influence on peak exercise capacity.

\section{S5 IDIOPATHIC PULMONARY ARTERIAL HYPERTENSION DEMONSTRATES A PERIPHERAL BLOOD SIGNATURE OF DYSREGULATED IMMUNITY}

${ }^{1} \mathrm{~K}$ Zalewska, ${ }^{2} \mathrm{E}$ Groves, ${ }^{1} \mathrm{H}$ Baxendale, ${ }^{1} \mathrm{M}$ Southwood, ${ }^{1} \mathrm{~J}$ Pepke Zaba, ${ }^{2} \mathrm{~N}$ Morrell, ${ }^{1} \mathrm{M}$ Toshner. ${ }^{1}$ Papworth Hospital NHS Foundation Trust, Cambridge, UK; ${ }^{2}$ Cambridge University, Cambridge, UK

\subsection{6/thoraxjnl-2015-207770.11}

Introduction There is increasing evidence of an association between Idiopathic Pulmonary Arterial Hypertension (IPAH) and immune dysregulation. Amongst other evidence, autoantibodies are detected in IPAH and there is a high prevalence of autoimmune conditions associated with group 1 pulmonary arterial hypertension. ${ }^{1}$ Previous work has focussed on a role for T-regulatory cells in IPAH, however, we have noted a more profound general lymphopaenia clinically, which we set out to define and investigate.

Objectives To phenotype circulating leucocytes in patients with IPAH.

Methods Fresh peripheral blood leucocytes from patients with IPAH were compared to age and sex-matched healthy controls. A standardised flow cytometry panel for cell surface markers of leucocyte sub-populations was adapted from the Human Immunology Project. ${ }^{2}$ Additionally Ig subclasses in serum were analysed by PEG enhanced immunoturbidometric assay and nephelometry. Peripheral lung tissue from explants in patients with IPAH was compared to control tissue from lung tumour resection (distant to the tumour margin). Tissue was immunostained for complement fragments $\mathrm{C} 3 \mathrm{~d}$ and $\mathrm{C} 4 \mathrm{~d}$ by streptABC peroxidase technique and visualised with 3,39-DAB hydrochloride and imaged by light microscopy.

Results Overall lymphocyte counts were significantly reduced in IPAH compared to controls ( $p=0.0187)$. Despite this, IPAH patients demonstrated significantly increased populations of $\mathrm{T}$ follicular helper $(\mathrm{Tfh})$ cells $(\mathrm{p}=0.0007)$ and PD $1^{+} \mathrm{CD} 4^{+} \mathrm{T}$-cells $(\mathrm{p}=0.0028)$, associated with T-cell exhaustion and control of Tfh mediated humoral immunity. There was evidence of altered B-cell differentiation with increased transitional cells $(\mathrm{p}=0.01)$ and decreased non-switched memory cells $(\mathrm{p}=0.001)$. Ig subclasses were not statistically different between IPAH and control 